\title{
Sense disambiguation in polysemous words: cognitive perspective
}

\author{
Vera I. Zabotkina ${ }^{a}$, Elena L. Boyarskaya ${ }^{b}$ \\ ${ }^{a}$ Russian State University for the Humanities, Moscow, Russia \\ ${ }^{b}$ I.Kant Baltic Federal University, Kaliningrad, Russia
}

\begin{abstract}
In this paper the authors put polysemy research into the broader context of research into the mental lexicon, cognitive context conceptual priming, and probabilistic conceptual modelling. The article adopts a novel approach to the resolution of polysemy and puts it to an empirical test. The authors argue that priming plays a key role in the activation of an adequate meaning of a polysemous word. Mental structures, represented by a prime lexical unit, contain relevant conceptual information on the target word meaning. The prime triggers a cognitive context that influences the selection process of the target word sense.
\end{abstract}

Key words: mental lexicon, conceptual modelling, priming, polysemy, mental representations, sense disambiguation, cognitive contexts

\section{Introduction}

Resolving polysemy has always been and remains one of the key issues in both traditional semantics and cognitive semantics. In cognitive semantics, a polysemous word is interpreted as a mental structure resulting from the complex nature of human experience, and our understanding and interpretation of it. Language crystalizes reality, and shows how it is perceived, categorised and construed by the human mind. Every word is a result of certain cognitive processes (Kubryakova, 1997, p.38).

Polysemous words do not develop their senses at random, but "follow certain cognitive paths or patterns that are natural to human cognition and that structure our acquisition of experience, knowledge of the world and language" (Nerlich et alt., 2003, p.7). Language is understood as a cognitive human activity which draws "on patterns that are regulated in accordance with a community's sociocultural practices" (S.Cowley, 2011). Human knowledge of the world in all its complexity is stored in the mental lexicon.

We can argue that the mental lexicon performs an important role in polysemy resolution, since it is the mental lexicon that concentrates various types of cognitive 
processes connected with perception, processing, storage, retrieval, usage and generation of knowledge. There is no unanimously accepted understanding of what the mental lexicon is and what functions it performs. Opinions vary: some consider it to be a mental store of information about words that includes semantic information (words' meaning), syntactic information (how words are combined to form sentences, and the details of word forms.) Some theories present the mental lexicon as an instrument for both language comprehension and production, while other theories distinguish between input and output lexica (M.Gazzaniga et alt., 2009, pp.389-390). There are also opinions that the mental lexicon is organized in the form of information-specific networks. It is often understood as a system of concepts and links between them which have been formed as a result of human cognitive activity. However, there is a general understanding that the mental lexicon is an inherent part of human memory; it is directly connected with language and the role it plays in cognition. On the one hand, the mental lexicon is a kind of analogue of a certain natural language. On the other, it is an integral part of the human mind, intellect and human memory (Kubryakova, 2004, p.379).

Given that context is a key factor in resolving polysemy, the central question in the theory of polysemy is still that of what aspects of word meanings are predefined and invariant across multiple contexts, versus what other aspects are indeterminate and only realized in context (Ravin, Leacock, 2002, pp.5-6). The research presented in this article focuses on different types of cognitive contexts stored in the mental lexicon that are activated during the process of word sense disambiguation.

\section{Mental lexicon, cognitive contexts and polysemy}

The formation of the mental lexicon takes place throughout life. From the age of 16 months, children acquire 10 or more new words daily (Eysenk, Keane, 2010, p.328), and a normal adult speaker has a passive vocabulary of about 50000 words (M.Gazzaniga et alt., 2009, p.389). Bearing in mind that both passive and active vocabulary units are polysemous, polysemy resolution mechanisms never fail to attract attention. Multiple word senses are never a problem for human communication. Speakers unconsciously and quickly select the only polysemous word sense that suits a particular context. However, there is purposeful work of the brain behind every polysemy resolution task.

The method of conceptual modelling allows the authors to reconstruct the process of the formation of certain fragments of the mental lexicon related to perception, processing, storage and retrieval of the information connected with particular senses of a polysemous word.

Since most words in natural languages are polysemous, we may assume that every word has a complex mental representation associated with it. The complex mental representation is a result of the perception, decoding, processing, storage and retrieval of various types of information directly and indirectly, relating to the polysemous word. This complex mental representation is a combination of cognitive contexts which store various types of information on all types of situations of their usage, real or potential participants, their actions, the sequence of these actions, etc. Thus, a cognitive context is a mental phenomenon of a complex nature. Each 
meaning of a polysemous word has its own cognitive context; a combination of all cognitive contexts associated with all the meanings of a polysemous word creates a complex mental representation of the polysemous word.

Cognitive contexts are not static properties; they reflect the dynamic character of human cognition as such. They do not have a once-and-for-all given content, because their content is constantly adjusted in the process of our perception of reality, gaining new experience and assessing it. The acquisition of the senses of polysemous words progresses with varying intensity depending on the intensity of cognitive activity in general. A child first acquires the words denoting concepts belonging to the basic level of categorization. These words are more frequently used, and as research proves, are acquired more easily; they are also processed and identified faster.

All word senses (irrespective of the concepts they denote) are acquired and learned when used in a specific context. In our study the notion of cognitive context is understood broadly, and includes a whole range of cognitive context sublevels universal, culture-specific, individual, etc. in all the multitude of their parameters. As our empirical research shows, a broad cognitive context may contain 'universal' conceptual information which is often perceived and processed by default. This information is implicitly present in a given cognitive context, associated with a certain sense of a polysemous word. For instance, spatial relations (up-down, aboveunder), direction, physical structure of the world (e.g. "the sun rises in the east"), time (e.g. past-present-future), movement and its character, etc. Content-wise, this type of conceptual information is acquired in the course of ontological development, and reflects the ontological characteristics of human experience. This is the kind of conceptual information which is seldom (if ever) updated and will remain physically unchanged irrespective of any pragmatic factors; it is a certain conceptual constant that is always implicitly present. However, without this information, any cognitive context will be incomplete. Cognitive scenarios (or any other knowledge representation forms) represent typical, conventional situations, actions, their sequences, etc. and usually develop against a certain background. For instance, if we take the word 'menu' (which is polysemous), its cognitive scenario 'records' all the actions and their sequence associated with eating out - a waiter brings the menu, customers choose the dishes they want, etc. But the human mind registers not only and exclusively what the menu is and its telic function. It effortlessly perceives, processes and stores all the information associated with the concept menu - spatial organization of a restaurant, a café, other customers sitting at their tables, a head waiter meeting customers by the door, etc. This information (which can be initially perceived either consciously or by default) may play an important role and potentially generate new information by means of inference. In the case of polysemy, this type of cognitive context perceived by default may help to identify senses of the polysemous word. The following example shows how strongly this type of cognitive context may influence the word sense selection process, sometimes resulting in mistakes:

The pastor noticed little Alex staring at the large plaque that hung in the foyer of the church.

It was covered with names, and small American flags were mounted on either side of it. 
The seven year-old had been staring at the plaque for some time, so the pastor walked up, stood beside the boy, and said quietly, "Good morning, Alex."

"Good morning," replied the young man, still focused on the plaque. "What is this?" Alex asked.

"Well, son, it's a memorial to all the young men and women who died in the service."

Soberly, they stood together, staring at the large plaque. Alex's voice was trembling and barely audible when he asked, "Which service, the 9:45 or the 11:15? (http://www.emmitsburg.net)

The physical setting of the conversation (a church), an interlocutor (a pastor) and an activity (attending a morning service) obviously made the boy think of the only meaning of the word service - "a religious ceremony that takes place in a church". By the age of seven the boy must have heard the word service being used in a variety of meanings and contexts, starting with service in the senses "work done by people" and "turn to serve" in sports. However, the physical setting dominated the sense selection process, resulting in the misidentification of the word sense.

The culture-specific cognitive context includes information on the cultural specificities of the situation that the meaning of a polysemous word is associated with. This is the kind of knowledge that is characteristic of a particular community sharing the same physical and social environment. For instance, the meaning of the polysemous word number, used in sentences like "Please take a number", may be misinterpreted by people coming from countries where the system of queue numbers in shops or public institutions is not common. In this case an additional explanation is needed: "The ticket hall had one of those systems where you take a number from a machine by the door and wait for it to appear above one of the ticket windows" (British National Corpus). In this case the physical setting of the conversation is culture-specific.

Stored in human memory, cognitive contexts associated with particular senses of a polysemous word are the fruits of individual perception. Cognitive contexts associated with different senses of a polysemous word are inevitably subjective to a degree, since they are formed on the basis of our previous individual experience. Even when using a word in the same sense, two speakers, without realizing it, operate a different volume of conceptual information fixed by the same word sense. When perceiving, processing and storing a cognitive context associated with a particular polysemous word sense, every person has his/her own conceptual world view depending on his/her previous experience, and his/her subjective idea of the value scale formed during earlier life. This type of cognitive context reflects the epistemic characteristics of cognition.

Hence, each polysemous word is associated with a set of dynamic cognitive contexts forming a complex multi-dimensional mental representation, which could potentially capture and store a significant amount of conceptual information, referring in fact to any number of conceptual domains that are relevant to the identification of a particular sense of the word.

In our opinion the process of word sense acquisition and its storage in the mental lexicon can be formally represented in the following way:

- $\quad$ perception of the word (or a polysemous word sense); 
- holistic individual perception of the cognitive context associated with the word; visual and perceptual categorization at the conceptual pre-semantic stage;

- association of the word (or its particular sense) with a particular cognitive context;

- accumulation of cognitive contexts associated with different senses of the polysemous word in the process of the multiple use of the word in a variety of situations;

- formation of a complex mental representation (a set of cognitive contexts) and its storage in the mental lexicon.

Consequently, in the mental lexicon a polysemous word may be represented by a complex mental representation - a set of cognitive contexts associated with different senses of the polysemous word. This mental representation may 'record' a large volume of information belonging to different conceptual domains.

We also attempt to analyze the opposite process - the process of word retrieval from the mental lexicon, specifically polysemous word sense disambiguation, with the help of conceptual priming.

In a traditional semantic priming task, subjects are presented with pairs of words. The first member of the pair, the prime, is a word; the second member, the target, can be a real word, a non-word, or a pseudo-word. Subjects are faster and more accurate at making lexical decisions when the target word is preceded by a related prime, e.g. the prime 'car' for the target 'truck' (Gazzaniga et alt., 2009, p.390). So conceptual priming is a faster means of identifying a particular word meanly after the presentation of a prime belonging to the same conceptual category.

The results of our research show that a set of cognitive contexts (or a particular cognitive context) may act as a conceptual prime, leading to a faster and more accurate identification of the target word sense. Probabilistic conceptual modelling of word sense disambiguation clearly demonstrates the role of a particular type of cognitive context and conceptual primes in word sense disambiguation.

Conceptual modelling allows us to suggest a cognitive model simulating the process of resolving polysemy:

- $\quad$ perception of a polysemous word in one of its senses;

- activation of the complex mental representation (a set of cognitive contexts) associated with the word;

- activation of a particular cognitive context acting as a prime;

- word sense selection.

It should be noted that the sequence of conceptual operations presented above is only a hypothesis, and needs experimental evidence. However, it is still possible to illustrate the process of word sense disambiguation using data from the British National Corpus, specifically instances of new senses developing for polysemous English nouns. Let us take the word bird as an example, as we used it in our previous works on polysemy development and resolution (Zabotkina, Boyarskaya 2011, 2012).

The word bird has the following meanings recorded in most monolingual English dictionaries: 
n) warm-blooded egg-laying vertebrates characterized by feathers and forelimbs modified as wings

-S: (n) the flesh of a bird or fowl (wild or domestic) used as food

-S: (n) informal term for a (young) woman

-S: (n) a cry or noise made to express displeasure or contempt

-S: (n) badminton equipment consisting of a ball of cork or rubber with a crown of feathers

(WordNet)

Let us analyse the following sentences retrieved from the British National Corpus. We have chosen four groups of examples showing the use of the polysemous noun bird in some of its meanings:

(1) a. Glancing up, I saw a beautiful yellow bird perched on a telegraph wire, looking like a prize long-tailed canary.

b. The bird shuffled along the perch.

(2) a. Victoria Wood's quite an attractive bird for a fat lady.

b. Richie left with a bird yesterday.

c. Oh look ain't that, that same bird.

d. He had risen in the City to a financial position which permitted him to park his BMW beside the flash docklands flat, close to his rough but pure roots, in which he kept his nice French bird.

(3) a. How soon could Kirov get his bird off the ground, do you think?

b. That's a ${ }^{* * *}$ there is still no other bird that can take off!

(4) Ninety seven there it is now showing, Lot ninety seven, singing bird in a cage I have three hundred offered for this, three hundred pounds and twenty, three fifty, three eighty, four hundred and twenty, fifty four eighty five hundred fifty six hundred and fifty, seven hundred fifty eight hundred eight hundred pounds seated now, any more at eight hundred and fifty nine hundred and fifty nine fifty nine fifty, one thousand new bidder thousand one hundred one thousand one hundred pounds, any more?

In examples $1 \mathrm{a}$ and $1 \mathrm{~b}$ the word bird is used in its basic primary meaning. In 1a the cognitive context contains conceptual information which serves as a clue for the proper identification of the word sense - spatial position of the object (glancing up), location (perched on a telegraph wire), appearance (beautiful and yellow, looking like a prize long-tailed canary). Even if we had been given a minimum of conceptual information - "glancing up, I saw a beautiful yellow bird..." - it would not have been difficult to identify the meaning of the word since most of us have seen a rather familiar picture many times - birds sitting on telegraph wires. The cognitive context that is activated in 1a ('birds sitting on telegraph wires') helps to identify the precise meaning of the word bird.

In example $1 \mathrm{~b}$ the noun bird is also used in its basic meaning. However, the character and type of movement of the bird - "shuffled"- activate a cognitive context which is different from the cognitive context in example 1a. This cognitive context is also associatively connected with a bird, but a larger and heavier one, probably 
hardly able to fly (one of the prototypical characteristics of birds). The meaning of the word (as it is given in traditional dictionaries) is the same, but the volume of conceptual information and cognitive contexts associated with these senses are different. It makes us think about some domestic birds moving slowly along a perch.

In the second group of examples, cognitive contexts activate another meaning of the polysemous word belonging the category HUMAN BEING - a female (Victoria Wood, lady), having certain physical (fat) and evaluative (attractive) characteristics. The activation of these conceptual domains helps to identify the metaphorical and pragmatically marked meaning - "an attractive woman".

In $2 \mathrm{~b}$ a different cognitive context is activated. It contains information about a rather stereotypical situation - a man leaves a restaurant/bar/party accompanied by a young and attractive girl. This cognitive context is so stereotypical that any part of it can be easily reconstructed. We have seen it happen many times in real life, in movies, etc. We can even reconstruct actions/events preceding the ones described in the sentence. This previously acquired cognitive context helps to identify the metaphorical meaning of the word bird.

In example $2 \mathrm{c}$ the purely linguistic context is so narrow that it is impossible to identify the meaning of the polysemous word. However, a wider pragmatic context (a young man of 19 talks to his peers) and phonetic peculiarities of his speech make us think of the only target meaning - 'a young and attractive girl.' Our memory stores the information about young men of a particular age, their behaviour, topics of conversation, etc. We know from our previous experience what young boys are like, what they tend to speak about, etc. Consequently, a particular cognitive context, representing conceptual information and containing a number of pragmatic stratification characteristics, acts as a prime, helping to identify the sense of the target word - "an attractive girl".

The cognitive context in $2 \mathrm{~d}$ contains fairly stereotypical information about a well-off clerk, who has a prestigious and well paid job, "had risen in the City to a financial position", has an expensive apartment - a "flash docklands flat" - and an expensive car - "his BMW", as well as a pretty girlfriend who is French. This cognitive context is not culture-specific, it is so ubiquitous that it activates the only possible meaning - "a young and beautiful girl". To some extent, the resolution of polysemy here is very much expectancy-induced. For instance, if we ask anybody to continue the following sentence "He is an oligarch; he has a mansion, a Mercedes and...", then most people would probably add something like "a yacht, a ..., a wife and a lover". This stereotype was formed by the mass media. So when we hear a sentence like the one analyzed previously, our minds offer a cognitive context which evokes this stereotype.

In examples $3 \mathrm{a}$ and $3 \mathrm{~b}$ the noun bird is used in its metaphorical meaning - $\mathrm{a}$ plane. The complex mental representation in example 3a contains different types of conceptual information - on historical epoch (Kirov), place (Russia), action (get smth. off the ground). Being activated, these cognitive contexts taken together help to identify the metaphorical meaning of the word bird which can be found only in some dictionaries - a plane, missile, etc. Our background knowledge about the time of the action and our approximate assessment of the level of technology of that time narrow the volume of conceptual information of the target word to the sense 'plane', since missiles had not been designed at that time. 
In example $3 \mathrm{~b}$ a different cognitive context is activated - an airport, the impossibility of taking a flight (take off), irritation and anger (obscene words). A stereotypical situation, familiar to everybody who has had the experience of preparing to take a flight which is then cancelled due to technical problems or adverse weather conditions. Once experienced or perceived, this activated cognitive context may act as a prime, helping to identify the only sense possible - "a plane".

Example 4 shows another cognitive context - that of an auction. This cognitive context contains conceptual information about all the necessary elements of any auction - a seller (auctioneer), buyers (bidders), money (offered sums) and, finally the lot being sold - 'a bird in a cage'. The activated cognitive context makes it clear that the lot is not a live bird, since our stereotypical idea of an auction is not primarily associated with selling live birds, but rather works of art. So it is quite clear that "bird in a cage" is either a painting, or a statuette. Since there is no further information it is impossible to identify the sense more precisely, and the sentence remains vague.

\section{Conclusion}

Thus, we argue that every sense of a polysemous word has a set of cognitive contexts associated with it. The total of cognitive contexts of all word senses forms a complex mental representation. Cognitive contexts as inherent parts of the mental lexicon may perform the function of a prime, resulting in an easier and more precise identification of a polysemous word sense. However, further research is needed regarding the sequence of cognitive processes involved in polysemy resolution.

\section{References}

Distributed Language (2011). Ed. by Stephen J. Cowley, University of Hertfordshire, UK, John Benjamins Publishing Company.

Eysenk, V.W., Keane, M.T. (2010). Cognitive psychology. $6^{\text {th }}$ edition. New York: Psychology Press. Gazzaniga, M., Ivry, R., Mangun, G. (2009). Cognitive Neuroscience: the Biology of Mind. New York-London: W.W. Norton\&Company.

Kubryakova, E. (1997). Chasti rechi s kognitivnoi tochki zreniia [Parts of speech from the cognitive point of view]. Moscow.

Kubryakova, E. (2004). Jazik I znaniye: chasti rechi s kognitivnoi tochki zreniia. Rol' yazika v poznanii mira [Language and knowledge: parts of speech from the cognitive point of view. The role of language in the cognition of the world]. Moscow: the Languages of Slavic science.

Nerlich, B., Clarke, D. (2003). Polysemy and flexibility: an introduction and overview. In: Polysemy: Flexible Patterns of Meaning in Mind and Language. Trends in Linguistics: Studies and Monographs (p. 142). Berlin-New York: Mouton de Gruyer. doi: 10.1515/9783110895698.3

Zabotkina, V., Boyarskaya, E. (2011). Rol' praiminga v identificatii znachenia mnogoznachnogo slova [The role of priming in word sense disambiguation]. Materialy kruglogo stola "Vzaimodeistvie kognitivnih i jazikovih structur [Proceedings of the round table "Interaction of cognitive and language structures"]. Tambov: Russian Academy of Sciences. 